\title{
CD300c is an Activating Receptor Expressed on Human Monocytes
}

\author{
Venkateswara R. Simhadri John L. Mariano Aleksandra Gil-Krzewska \\ Qing Zhou Francisco Borrego \\ Laboratory of Molecular and Developmental Immunology, Division of Monoclonal Antibodies, Office \\ of Biotechnology Products, Center for Drug Evaluation and Research, Food and Drug Administration, \\ Bethesda, Md., USA
}

\author{
Key Words \\ CD300 $\cdot$ CD300a $\cdot$ CD300c $\cdot$ Cell surface receptors $\cdot$ Paired \\ receptors
}

\begin{abstract}
Human CD300 molecules comprise a family of receptors that regulate many immune cell processes. They are mostly expressed on myeloid cells, although expression of two members, CD300a and CD300c, has also been described on lymphocytes. However, due to the lack of specific antibodies that distinguish between these two receptors, it has been difficult to determine the expression pattern and function of CD300a and CD300c in primary cells. Here, we have identified a specific monoclonal antibody, clone TX45, that recognizes only CD300c and show that within freshly isolated blood leukocytes, monocytes are the only cells that express CD300c on the cell surface. In vitro differentiation experiments revealed that CD300c is differentially expressed on different monocyte-derived cells, including macrophages and dendritic cells. Furthermore, TLR ligands LPS and flagellin dynamically regulate the expression of CD300c. Crosslinking of this receptor with clone TX45 monoclonal antibody induced calcium mobilization, upregulation of the costimulatory molecule CD86 and the production of inflammatory cytokines. Importantly, LPS-mediated production of inflammatory cytokines by monocytes was further enhanced if CD300c was simultaneously engaged by the ag-
\end{abstract}

\section{KARGER}

E-Mail karger@karger.com

www.karger.com/jin onist antibody. Altogether, our results show that human CD300c is an activating receptor expressed on monocytes and that it has a potential role in inflammatory responses.

Copyright $\odot 2013$ S. Karger AG, Basel

\section{Introduction}

Myeloid cells are main sentinels of the immune system that have a prominent role in the host defense by sensing and monitoring foreign antigens $[1,2]$. Many of the myeloid functions are regulated through a set of cell surface molecules belonging to multigenic families that include both activating and inhibitory receptors, which in the end establish and maintain a delicate balance aimed to eliminate the foreign antigen while preserving self $[3,4]$. In general, the activating receptors of these multigenic families have charged residues in the transmembrane region and associate with adaptor molecules containing ITAM (immunoreceptor tyrosine-based activating motif) or phosphatidylinositol 3-kinases binding motif YxxM. After receptor engagement, ITAM and YxxM motifs become tyrosine phosphorylated and recruit protein kinases, initiating a downstream activation signaling cascade that regulates many functions such as cytokine secretion, calcium mobilization, differentiation, proliferation, phagocytosis, cytotoxicity and cell survival $[5,6]$. On the contrary, the inhibitory receptors have one or more ITIM
(C) 2013 S. Karger AG, Basel

$1662-811 \mathrm{X} / 13 / 0054-0389 \$ 38.00 / 0$
Dr. Francisco Borrego

Laboratory of Molecular and Developmental Immunology

Division of Monoclonal Antibodies, OBP, CDER, FDA, Building 29B, Room 3NN18 29 Lincoln Drive, HFD-123, Bethesda, MD 20892 (USA)

E-Mail Francisco.Borrego@fda.hhs.gov, francisco.borregorabasco@osakidetza.net 
(immunoreceptor tyrosine-based inhibitory motifs ) in their cytoplasmic tails that, after engagement by their ligands, also become tyrosine phosphorylated and serve as docking sites for phosphatase recruitment and its subsequent activation. Phosphatase activation initiates an inhibitory signaling cascade that downregulates activation signals $[7,8]$.

One such large family of leukocyte membrane receptors is the CD300 family of IgV-like glycoproteins that regulate immune responses via their paired activating and inhibitory receptors [9]. Recently, several publications have shown that members of the human and mouse CD300 families of receptors are able to recognize lipids and other ligands, and it has been reported that they have a very important role in regulating a variety of immunemediated processes [9-15]. The human activating members of this family include CD300b, CD300c, CD300d and CD300e, and associate with adaptor molecules such as FcR $\gamma$ chain (FceRI $\gamma$ ), DAP (DNAX-activating protein) 12 or DAP10 [9, 16-19]. The inhibitory receptors include CD300a and CD300f $[9,20]$. Phylogenetic analyses have shown that CD300a and CD300c are paired receptors with a homology in their extracellular immunoglobulin domain higher than $80 \%[21,22]$. Human CD300a has a long cytoplasmic tail with three classical ITIMs and one nonclassical ITIM that, after phosphorylation, associates with SHP-1, SHP-2 and SHIP phosphatases and elicits a signal that is capable of inhibiting the functions of natural killer (NK) cells, mast cells, B and T lymphocytes, macrophages, eosinophils, basophils, neutrophils and dendritic cells (DC) $[9,14,22-28]$. In contrast, CD300c has a short cytoplasmic tail, a negatively charged amino acid residue in the transmembrane region and the potential to associate with the adaptor molecules FceRI $\gamma$ and DAP12 [17, 18]. Although human CD300c was the first molecule to be identified in this family of receptors [29], its function on primary cells has not been elucidated so far.

Ligation of ectopically expressed human CD300c induces activating signals in the mast cell line RBL-2H3, partially through its direct association with FceRI $\gamma$ [18]. Nonetheless, its role in human cells that endogenously express the receptor is not known. Although transcripts encoding both CD300a and CD300c are widely found in both lymphoid and myeloid cell lineages [22, 26, 29, 30], the cell surface expression of these receptors has been difficult to discriminate. This is due to the lack of specific monoclonal antibodies that could differentiate between the surface expression of CD300a and CD300c [22, 26, 28, 30]. In this study, using an anti-CD300c-specific antibody, we describe the cell surface expression of CD300c in peripheral blood monocytes and its ability to induce activating signals. Contrary to CD300a, we show that $\mathrm{CD} 300 \mathrm{c}$ is not expressed on the surface of the vast majority of freshly isolated lymphocytes or granulocytes. In addition, we also show that $\mathrm{CD} 300 \mathrm{c}$ is regulated on monocytes after treatment with the TLR4 and TLR5 ligands LPS and flagellin, respectively, and that it is differentially expressed on monocyte-derived macrophages and monocyte-derived DC (mDC). Furthermore, our data also suggest that CD300c has a costimulatory effect in cytokine secretion on LPS-stimulated monocytes. Altogether, our results support the notion that $\mathrm{CD} 300 \mathrm{c}$ is an activating receptor in human monocytes and that it may have a significant role in regulating inflammatory responses.

\section{Materials and Methods}

\section{Study Population}

Whole-blood and buffy coats were collected under an institutional review board-approved protocol at the National Institutes of Health Blood Bank from healthy donors. All study subjects provided written informed consent.

\section{Constructs and 293T and YTS Cell Transfectants}

RNA was isolated from freshly isolated human NK cells using the RNAqueous-4PCR kit (Ambion-Life Technologies) and transcribed into cDNA using qScript cDNA synthesis kit (Quanta Biosciences). The full-length cDNAs corresponding to CD300a and CD300c were amplified using specific primers and the products were cloned into pcDNA3.1 (+) expression vector (Invitrogen) using standard molecular biology techniques. The constructs were sequenced to confirm their identity. Transient transfection of the constructs into $293 \mathrm{~T}$ cells, a human fibroblast kidney cell line, was performed using Lipofectamine 2000 according to the manufacturer's protocol (Invitrogen). YTS stable transfectants were obtained initially by electroporation, then sorted for the positive cells and finally selected in culture medium containing $1 \mathrm{mg} / \mathrm{ml}$ of gentamicin (InvivoGen).

Isolation of Peripheral Blood Nucleated Cells, Monocytes and Generation of Monocyte-Derived Cells

The human nucleated cell fraction was isolated from whole blood with HetaSep (Stem Cell Technologies) using either the gravity sedimentation or the centrifugation method according to the manufacturer's protocol. Enriched human monocytes were obtained from buffy coats of healthy donors using RosetteSep (Stem Cell Technologies), a negative selection method that depletes the unwanted cells with tetrameric antibody complexes recognizing CD2, CD3, CD8, CD19, CD56, CD66b, CD123 and glycophorin A. The purity of monocytes was more than $70 \%$. Macrophages and immature $\mathrm{mDC}$ were differentiated from enriched monocytes in differentiation media in the presence of recombinant human M-CSF (50 ng/ml) and recombinant human GM-CSF (50 ng/ml) plus recombinant human IL-4 $(20 \mathrm{ng} / \mathrm{ml})$, respectively, for 6 days. The composition of differentiation media was Iscove's modified Dulbecco's medium (plus GlutaMax) sup- 
plemented with $10 \%$ human $\mathrm{AB}$ serum, 2 mM glutamine, $1 \%$ sodium pyruvate and $1 \%$ non-essential amino acids (Lonza). The medium was replaced 2-3 times during the differentiation period. After 6 days of culture, the immature $\mathrm{mDC}$ were further cultured in the presence of $1 \mathrm{ng} / \mathrm{ml}$ LPS (InvivoGen) for $48 \mathrm{~h}$ to generate mature $\mathrm{mDC}$.

\section{Flow-Cytometric Analysis}

The following mouse anti-human antibodies were used for flow-cytometric analysis: purified and PE anti-CD300a/c IRp60 (clone E59.126) from Beckman Coulter; purified anti-CD300a/c (clone TX49) was a generous gift from Dr. Akira Shibuya; eFluor 660 anti-CD300c (clone TX45), PE-Cy7 anti-CD19 (clone H1B19), AF700 anti-CD3 (UCHT1) and PE-Cy7 anti-CD14 (clone 61D3) from eBioscience; FITC anti-CD66b (clone G10F5), purified and $\mathrm{PE}$ anti-CD300c (clone TX45) from Biolegend; PE anti-CD80 (clone L307.4), FITC anti-CD86 (clone 2331 FUN-1), PE antiCD83 (clone HB15e), PE anti-CD206 (clone 19.2), APC antiCD209 (clone DCN46) and APC anti-CD56 (clone NCAM16.2) from BD Biosciences. Purified and fluorochrome conjugated MOPC-21 (mouse IgG1; from eBioscience) was used as an isotypematched control. Secondary antibodies were purchased from Jackson ImmunoResearch. YTS cells stably expressing CD300a or CD300c were washed with staining buffer $(1 \times$ phosphate-buffered saline, PBS, plus $1 \%$ fetal bovine serum, FBS) and stained with the respective fluorochrome conjugated antibodies for $30 \mathrm{~min}$ on ice. Then, cells were washed to remove unbound antibodies and further acquired in a FACSCalibur flow cytometer (BD Biosciences). Regarding 293T cells transiently transfected with CD300a or CD300c encoding constructs, the cells were harvested after 16-20 $\mathrm{h}$ of transfection, washed with staining buffer and incubated with fluorochrome conjugated anti-CD300a/c (clone E59.126) or antiCD300c (clone TX45) monoclonal antibody for $30 \mathrm{~min}$ on ice. The 293T cells were also stained with purified anti-CD300a/c (clone TX49) for $40 \mathrm{~min}$, washed and incubated with secondary PE conjugated anti-mouse Fc antibody for another 20 min on ice. Then, cells were washed thoroughly and acquired in a FACSCalibur flow cytometer. For the flow-cytometric experiments with human primary cells, the staining buffer contains $1 \%$ human $A B$ serum to block the Fc receptors. Before harvest from the plates, monocytes and monocyte-derived macrophages were incubated with Accutase (Innovative Cell Technologies) for 15-20 min at room temperature to detach the cells. After harvesting, cells were washed in staining buffer and incubated with the respective fluorochrome conjugated antibodies for $30 \mathrm{~min}$ on ice. After extensive washing, the cells were then acquired using either FACSCalibur or LSRII (BD Biosciences) flow cytometers. Flow-cytometric data were analyzed using FlowJo software (Tree Star).

\section{Stimulation of Monocytes with LPS and Flagellin}

Enriched monocytes $\left(10^{6} / \mathrm{ml}\right)$ from healthy donors were either stimulated with LPS $(1 \mathrm{ng} / \mathrm{ml})$ or flagellin $(100 \mathrm{ng} / \mathrm{ml})$ in a $24-$ well plate for different time periods as mentioned in the text and figure legends. Then, cells were harvested and analyzed for CD300c surface expression by flow-cytometric analysis. In addition, cell samples for RNA isolation were stored at $4^{\circ} \mathrm{C}$ in RNAlater (AmbionLife Technologies). Real-time quantitative PCR assays were performed on a CFX-96 real-time PCR detection system (Bio-Rad) using 480 SYBR Green I master supermix (Quanta Biosciences) according to the manufacturer's protocol. Primers for real-time
PCR measurement of human CD300c (PPH07153A) and $\beta$-actin (PPH00073G) were purchased from SA Biosciences. All reactions were made in triplicate, and averages were used to calculate the relative levels of mRNA. Relative quantification of CD300c mRNA was determined using the second derivative maximum using the CFX-Manager software (Bio-Rad), and data were normalized to the housekeeping gene $\beta$-actin.

\section{Cross-Linking of CD300c on Monocytes and Measurement of} Cytokine Production

Tissue culture plates (24 wells) were coated with $10 \mu \mathrm{g} / \mathrm{ml}$ of either purified anti-human $\mathrm{CD} 300 \mathrm{c}$ monoclonal antibody (clone TX45) or the isotype control (MOPC-21) for $2-3 \mathrm{~h}$ at $37^{\circ} \mathrm{C}$ and then extensively washed with PBS to remove the unbound antibodies. Enriched monocytes $\left(10^{6} / \mathrm{ml}\right)$ obtained from healthy donors were then added to the antibody-coated plates in RPMI 1640 medium supplemented with $10 \% \mathrm{FBS}, 100 \mathrm{U} / \mathrm{ml}$ penicillin-streptomycin, $2 \mathrm{mM}$ glutamine and $10 \mathrm{mM}$ HEPES (Lonza). In the costimulation experiments, along with the plate-bound antibody activation, monocytes were also stimulated with the addition of LPS $(1 \mathrm{ng} / \mathrm{ml})$ to the culture. After $24 \mathrm{~h}$ of stimulation, the cell culture supernatants were harvested and stored at $-80^{\circ} \mathrm{C}$, and the cells were harvested and analyzed by flow cytometry for the expression of costimulatory molecules.

The cell culture supernatants were thawed on ice and analyzed for cytokine production using the human inflammatory cytokine kit by BD Biosciences cytometric bead arrays according to the manufacturer's protocol. Samples were acquired on a BD LSRII flow cytometer (BD Biosciences) and analyzed using BD FCAP array software.

\section{Calcium Mobilization Assay}

Freshly isolated monocytes were washed and resuspended in Dulbecco's PBS (GIBCO catalog No. 14287) containing 1\% bovine serum albumin at $5 \times 10^{6}$ cells $/ \mathrm{ml}$. Next, cells were labeled with Fura Red $(5 \mu \mathrm{g} / \mathrm{ml})$ and Fluo-4 $(2 \mu \mathrm{g} / \mathrm{ml})$, from Invitrogen, for $30 \mathrm{~min}$ at $30^{\circ} \mathrm{C}$. Then, cells were washed twice and resuspended at $2 \times 10^{6}$ cells $/ \mathrm{ml}$. Aliquots of $1 \mathrm{ml}$ were warmed at $37^{\circ} \mathrm{C}$ for $5 \mathrm{~min}$, followed by acquisition in a FACSCalibur flow cytometer. To establish a baseline, cells were first acquired for $30 \mathrm{~s}$ and then they were cross-linked either with $5 \mu \mathrm{g}$ of anti-CD300c (clone TX45) or isotype control (MOPC-21) for $30 \mathrm{~s}$, followed by the addition of $8.5 \mu$ goat anti-mouse $\operatorname{IgG~F}\left(\mathrm{ab}^{\prime}\right)_{2}$. Cells were further acquired for 6 min. Data were analyzed using the FlowJo software (Treestar).

\section{Statistical Analysis}

Data were analyzed using GraphPad Prism software. The data were plotted as bar graphs, and pairwise comparisons were examined by a paired Student's t test. ${ }^{*} \mathrm{p}<0.05,{ }^{* *} \mathrm{p}<0.01$.

\section{Results}

\section{Specific Recognition of Human CD300c by the} Monoclonal Antibody Clone TX45

Human CD300a and CD300c mRNA transcripts are broadly detected in cells from both the myeloid and lymphoid lineages $[22,26,29,30]$. However, the cell surface 


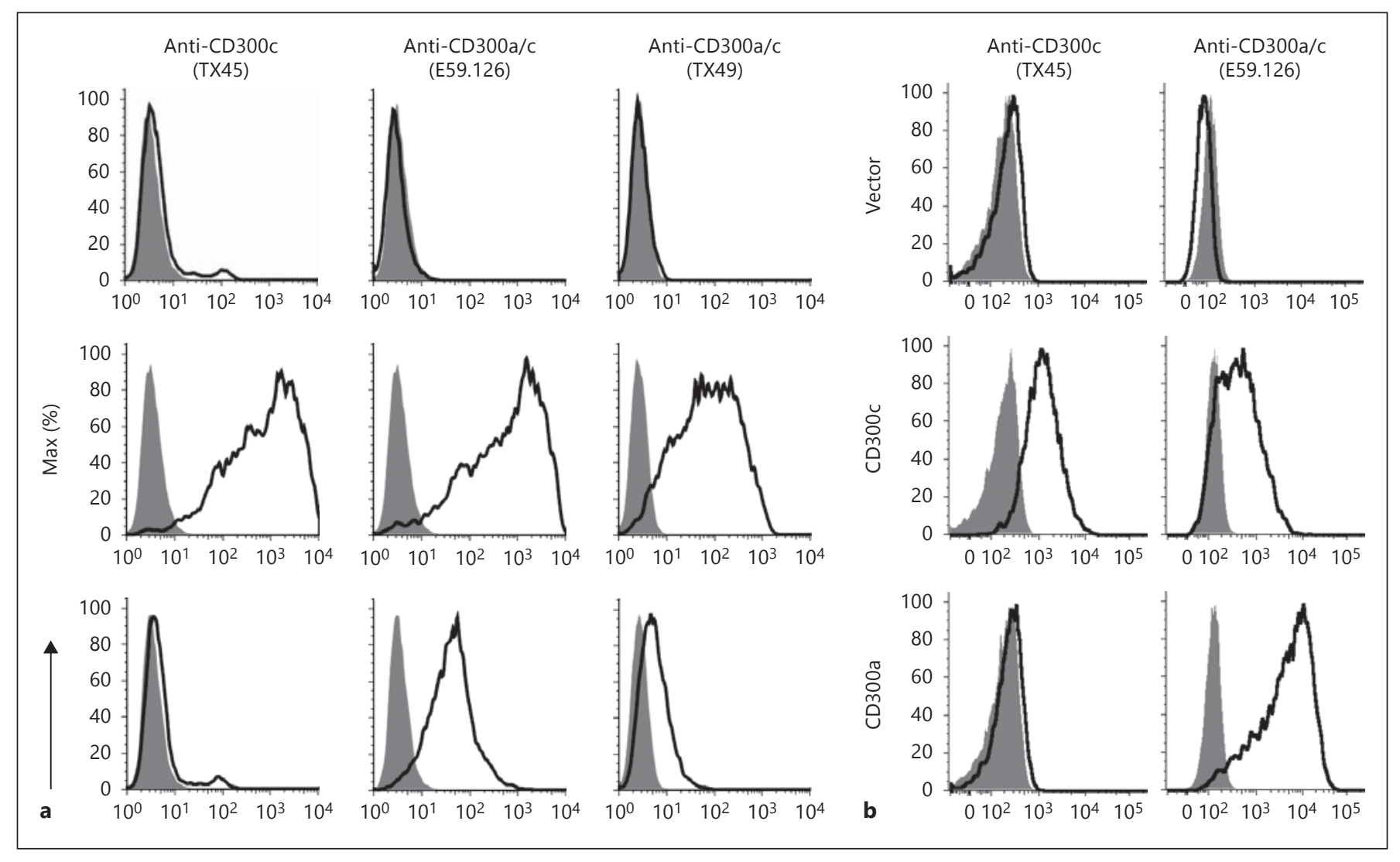

Fig. 1. Monoclonal antibody clone TX45 binds specifically to CD300c. a 293T cells were transiently transfected with empty vector (upper panels), vectors expressing full-length CD300c (middle panels) or CD300a (lower panels). After $16 \mathrm{~h}$ of transfection, cells were stained with the following monoclonal antibodies: antiCD300c, clone TX45 (left lane); anti-CD300a/c, clone E59.126 (middle lane), and anti-CD300a/c, clone TX49 (right lane). Shaded histograms represent unstained cells and the open histograms represent the staining with the specific antibodies. These data are rep-

expression of these two highly homologous receptors has been difficult to determine because of the lack of specific antibodies that are able to discriminate between CD300a and CD300c. The majority of available antibodies show cross-reactivity and recognize both receptors on the cell surface $[22,28,30]$. Thus, it has been a very difficult, if not an impossible task to investigate and understand the specific role of these two molecules in primary cells.

As a first step, we wanted to examine if there is a monoclonal antibody that binds specifically to cell surface CD300c but not to CD300a. Plasmids encoding full-length $\mathrm{CD} 300 \mathrm{a}$ and $\mathrm{CD} 300 \mathrm{c}$ were generated and transfected into 293T and YTS cells. After testing several antibodies, we found that clone TX45, a commercially available monoclonal antibody, did not bind to $293 \mathrm{~T}$ cells transfected resentative of 3 independent experiments. b YTS cells stably transfected with empty vector (upper panel), vector expressing CD300c (middle panel) and CD300a (lower panel) were stained with antiCD300c, clone TX45 (left lane) and anti-CD300a/c, clone E59.126 (middle lane). The shaded histograms represent the isotypematched controls and the open histograms the staining with the specific monoclonal antibodies. Results are representative of 3 independent experiments.

with either empty vector control or with a construct encoding CD300a. However, clone TX45 showed a strong binding to CD300c transfected cells (fig. 1a). On the contrary, the monoclonal antibodies clones E59.126 and TX49 did recognize transfectants expressing both CD300a and $\mathrm{CD} 300 \mathrm{c}$, indicating that these two antibodies are not specific (fig. 1a). In addition, we wanted to test the specificity of clone TX45 on lymphoid cells expressing CD300a and CD300c. To perform that, we generated CD300a or CD300c stable transfectants of YTS, an NK cell tumor cell line. Similar to the results mentioned with the 293T cells, clone TX45 bound only to CD300c-expressing YTS cells and clone E59.126 bound to both CD300a and CD300c transfected YTS cells. As expected, none of these antibodies showed any binding to the empty vector transfected 
YTS cells (fig. 1b). Thus, despite the very high homology between the extracellular domains of CD300a and CD300c, we show that the anti-CD300c monoclonal antibody, i.e. clone TX45, binds specifically to human CD300c and does not cross react with $\mathrm{CD} 300 \mathrm{a}$.

CD300c is Expressed on the Surface of Monocytes and Monocyte-Derived Cells

The identification of a specific monoclonal antibody against CD300c, clone TX45, was of great interest, and therefore we decided to study the cell surface expression of this receptor on cells of both lymphoid and myeloid lineages. The nucleated cell fraction was isolated from the whole blood of healthy donors and the binding of the monoclonal antibodies anti-CD300a/c, clone E59.126, and anti-CD300c, clone TX45, to different cell subsets was tested. We confirmed previously published results $[23,27,28,31,32]$ and showed that clone E59.126 binds to all NK cells (CD3-CD56+), monocytes (CD14+), granulocytes $(\mathrm{CD} 66 \mathrm{~b}+)$ and subsets of $\mathrm{T}(\mathrm{CD} 3+)$ and $\mathrm{B}$ cells (CD19+; fig. 2a). Intriguingly, the specific monoclonal antibody against CD300c, clone TX45, binds almost exclusively to the CD14+ population and not to any other cell type (fig. 2a). In some donors, a very small subset of $\mathrm{T}$ cells, less than $2 \%$, bound clone TX45 (data not shown). These results indicate that CD300a and CD300c are differentially expressed. In freshly isolated peripheral blood cells, CD300a is expressed on the cell surface of lymphocytes, monocytes and granulocytes, while CD300c is almost exclusively expressed on monocytes.

We then enriched monocytes from the peripheral blood using negative selection and in vitro generated mDC and macrophages. Phenotypic analysis confirmed the purity of the different cell types: monocytes (CD14+), macrophages (CD14 ${ }^{\text {high }} \mathrm{CD} 206++\mathrm{CD} 83-\mathrm{CD} 80-$ CD86+), immature mDC (CD14 ${ }^{\text {low }}$ CD209+++ CD80+ CD86+ CD83-) and mature mDC (CD14 ${ }^{\text {low }} \mathrm{CD} 209+++$ CD80+++ CD86+++ CD83++; data not shown). Our data showed that monocyte-derived macrophages are positive for CD300c, with similar expression levels to those observed on peripheral blood monocytes. On the contrary, we observed that the cell surface expression of CD300c was reduced on immature and LPS-induced mature $\mathrm{mDC}$ relative to monocytes and macrophages (fig. 2b). Additionally, the anti-CD300a/c (clone E59.126) monoclonal antibody binds to all monocyte-derived cells without any change in the intensity of cell surface expression (data not shown). Thus, the surface expression of CD300c is exclusive to monocytes and monocyte-derived cells. Since it has been reported that $\mathrm{CD} 300 \mathrm{c}$ transcripts are found in other

CD300c: An Activating Receptor on Human Monocytes cell types, further studies are needed to understand the mechanism regulating the cell surface expression of this receptor.

\section{Expression of CD300c is Regulated on Monocytes upon LPS Treatment}

To further investigate how CD300c expression is regulated, we treated freshly isolated enriched monocytes with the TLR4 ligand LPS. Gene expression analysis showed that after 2-hour treatment with LPS, the levels of CD300c transcripts were significantly downregulated, while after $24 \mathrm{~h}$ of treatment the amount of $C D 300 \mathrm{c}$ transcripts increased to the levels expressed by nonstimulated monocytes (fig. 3a). On the other hand, although the cell surface expression of CD300c decreased after LPS treatment for 2 $\mathrm{h}$, in line with the amount of CD300c transcripts, after 24 $\mathrm{h}$ of LPS stimulation CD300c expression showed a significant increase when compared with untreated monocytes (fig. 3b). Similar results were observed when monocytes were treated with the TLR5 ligand flagellin (online supplfig. 1; for all online suppl. material, see www.karger.com/ doi/10.1159/000359523). Therefore, our results suggest that the regulation of $\mathrm{CD} 300 \mathrm{c}$ expression on monocytes is a highly dynamic process, probably involving both transcriptional and posttranscriptional mechanisms.

\section{Triggering of CD300c Induces Calcium Mobilization and Upregulation of CD86}

To characterize the function of $\mathrm{CD} 300 \mathrm{c}$ in circulating monocytes, we studied whether the anti-CD300c-specific monoclonal antibody clone TX45 is able to induce activation signals in monocytes. We first investigated its ability to induce intracellular calcium mobilization. Engagement of CD300c with soluble anti-CD300c monoclonal antibody followed by cross-linking with anti-mouse IgG $\mathrm{F}\left(\mathrm{ab}^{\prime}\right)_{2}$ induced a transient and rapid increase in intracellular calcium (fig. 4a), which was not observed when cells were stimulated under the same conditions with the isotype-matched control (clone MOPC-21). Engagement of CD300c only with soluble anti-CD300c monoclonal antibodies, in the absence of cross-linking with anti-mouse $\operatorname{IgG~} \mathrm{F}\left(\mathrm{ab}^{\prime}\right)_{2}$, did not induce calcium mobilization (data not shown). To further prove that $\mathrm{CD} 300 \mathrm{c}$ is an activating receptor, we stimulated freshly isolated monocytes with plate-bound anti-CD300c monoclonal antibody. After 24 $\mathrm{h}$ of culture, monocytes were able to significantly upregulate the cell surface expression of the costimulatory molecule CD86, while monocytes cultured on plates coated with the isotype-matched control did not show an increase in the expression of CD86 (fig. 4b). Interestingly, 


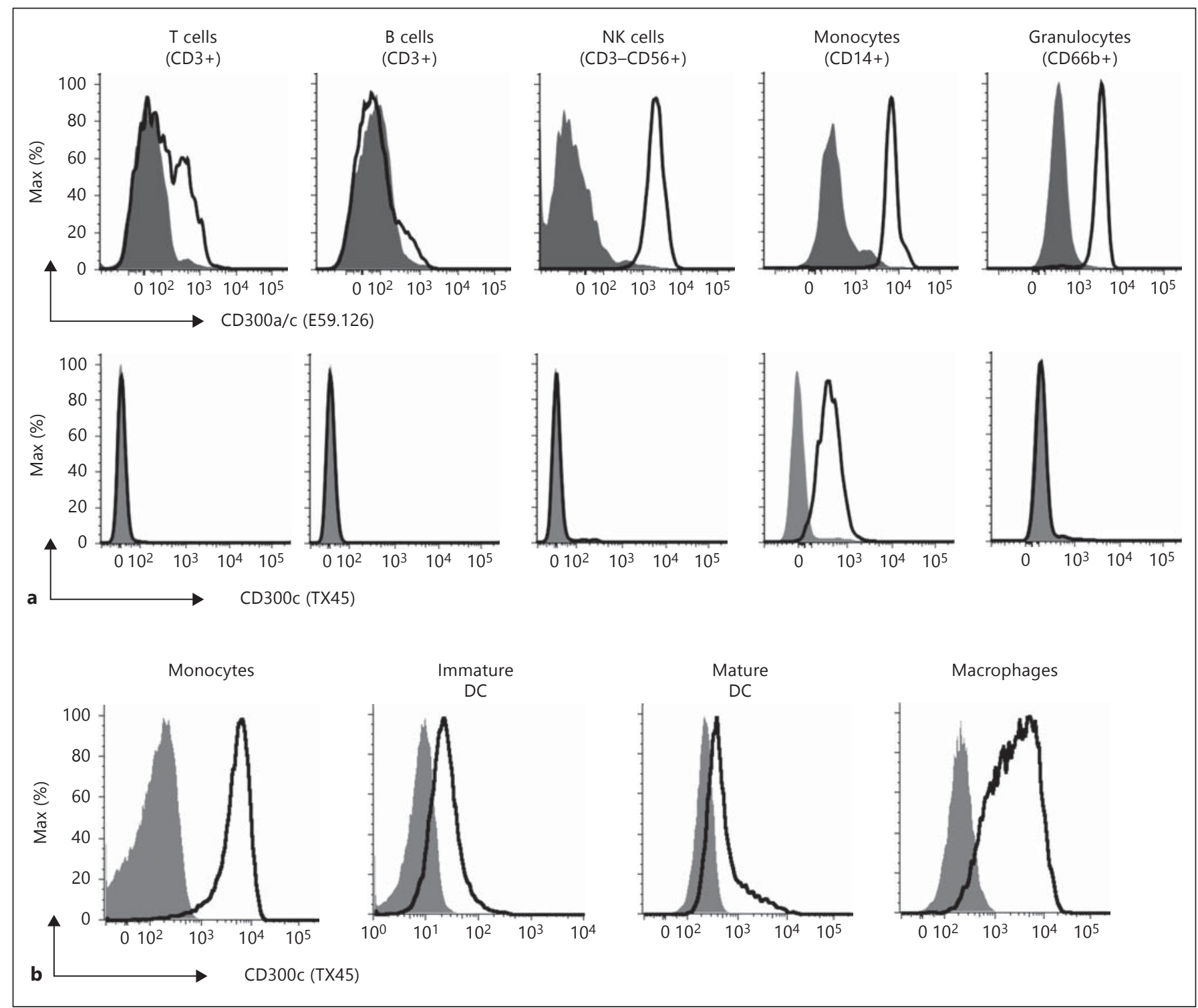

Fig. 2. CD300c is expressed on human monocytes and monocytederived cells. a Cells were isolated from normal healthy donors and stained with specific antibodies to distinguish different cell subsets. In addition, cells were stained with anti-CD300a/c (clone E59.126) and anti-CD300c (clone TX45) monoclonal antibodies. Shaded histograms represent the staining with isotype-matched controls and empty histograms the staining with anti-CD300a/c (clone E59.126; upper panel) and anti-CD300c (clone TX45; lower

in our experimental settings, no change was found in the cell surface expression of CD80 after stimulation of monocytes with plate-bound anti-CD300c monoclonal antibody (data not shown). Thus, our data support the notion that $\mathrm{CD} 300 \mathrm{c}$ is a functional activating receptor in monocytes. panel). Data from a representative out of 6 are shown. $\mathbf{b}$ Enriched monocytes were differentiated into $\mathrm{mDC}$ and macrophages according to the protocol described in the Materials and Methods. The expression of CD300c was determined using anti-CD300c (clone TX45) monoclonal antibody. The shaded histograms represent the staining with isotype-matched control and the open histograms correspond to the specific staining with anti-CD300c (clone TX45). Results from a representative out of 4 are shown.

\section{Cross-Linking of CD300c Upregulates \\ Proinflammatory Cytokine Secretion from LPS-Treated Monocytes}

As monocytes are very important players in the control of inflammatory processes, we investigated if $\mathrm{CD} 300 \mathrm{c}$ engagement has a role in the production of pro- 


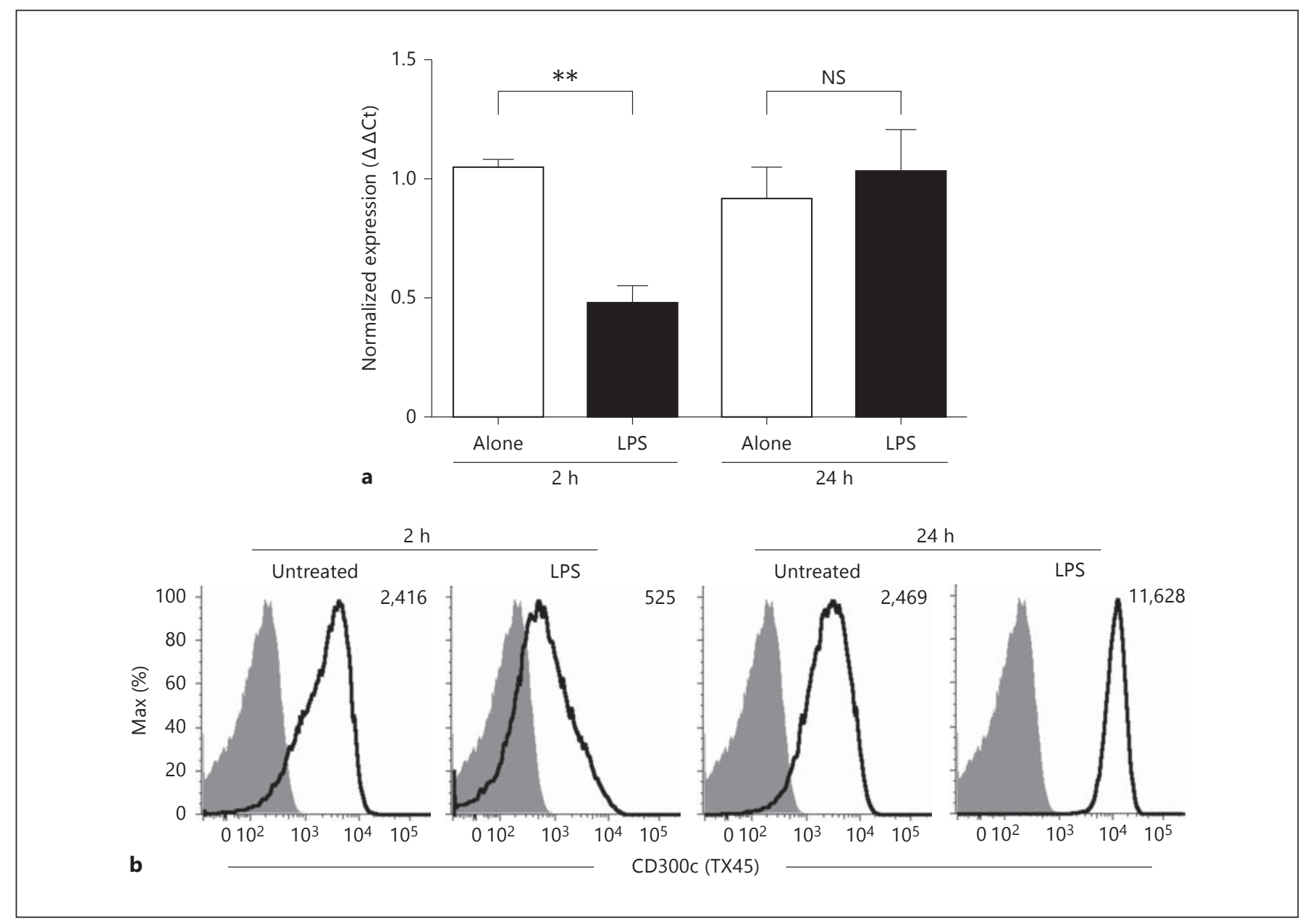

Fig. 3. Expression of CD300c is regulated on monocytes by LPS. a Enriched monocytes from healthy donors were either left untreated (Alone) or treated with LPS for 2 and $24 \mathrm{~h}$. RNA was extracted and the levels of $C D 300 c$ mRNA were determined by realtime PCR. Graph bars represent the average \pm SEM. Data are from 5 independent experiments. b Enriched monocytes were either left untreated or treated with LPS for 2 and $24 \mathrm{~h}$. The cells were

inflammatory cytokines. Freshly isolated monocytes were cultured either with plate-bound anti-CD300c monoclonal antibody (clone TX45) or with isotypematched control (clone MOPC-21). Cross-linking of CD300c induced significant production of the inflammatory cytokines TNF- $\alpha$ and IL-8, while there was not a significant increase in IL- 6 and IL- $1 \beta$ secretion compared with monocytes cultured with plate-bound isotype-matched control (fig. 5). TLRs are crucial in the recognition of pathogens, and their engagement on monocytes activates the secretion of pro- and anti-inflammatory cytokines [33]. We sought to determine if harvested and checked for CD300c cell surface expression. The shaded histograms represent the staining with the isotype control (MOPC-21) and the open histograms represent the staining with anti-CD300c (clone TX45) monoclonal antibody. The numbers indicate the values of median fluorescence intensity. Results are representative of 5 healthy donors. cross-linking of CD300c is able to modulate TLR-mediated cytokine production. Our results showed that CD300c engagement significantly increased LPS-mediated TNF- $\alpha$, IL- $1 \beta$, IL- 6 and IL-8 secretion (fig. 5). In our experimental conditions, no IL-12 was detected (data not shown). Intriguingly, the LPS-mediated secretion of the anti-inflammatory cytokine IL-10 did not increase when CD300c was cross-linked (fig. 5). Altogether, these data further point out that CD300c is a novel activating receptor expressed on human monocytes and that is able to cooperate with TLR4 in the production of inflammatory cytokines. 


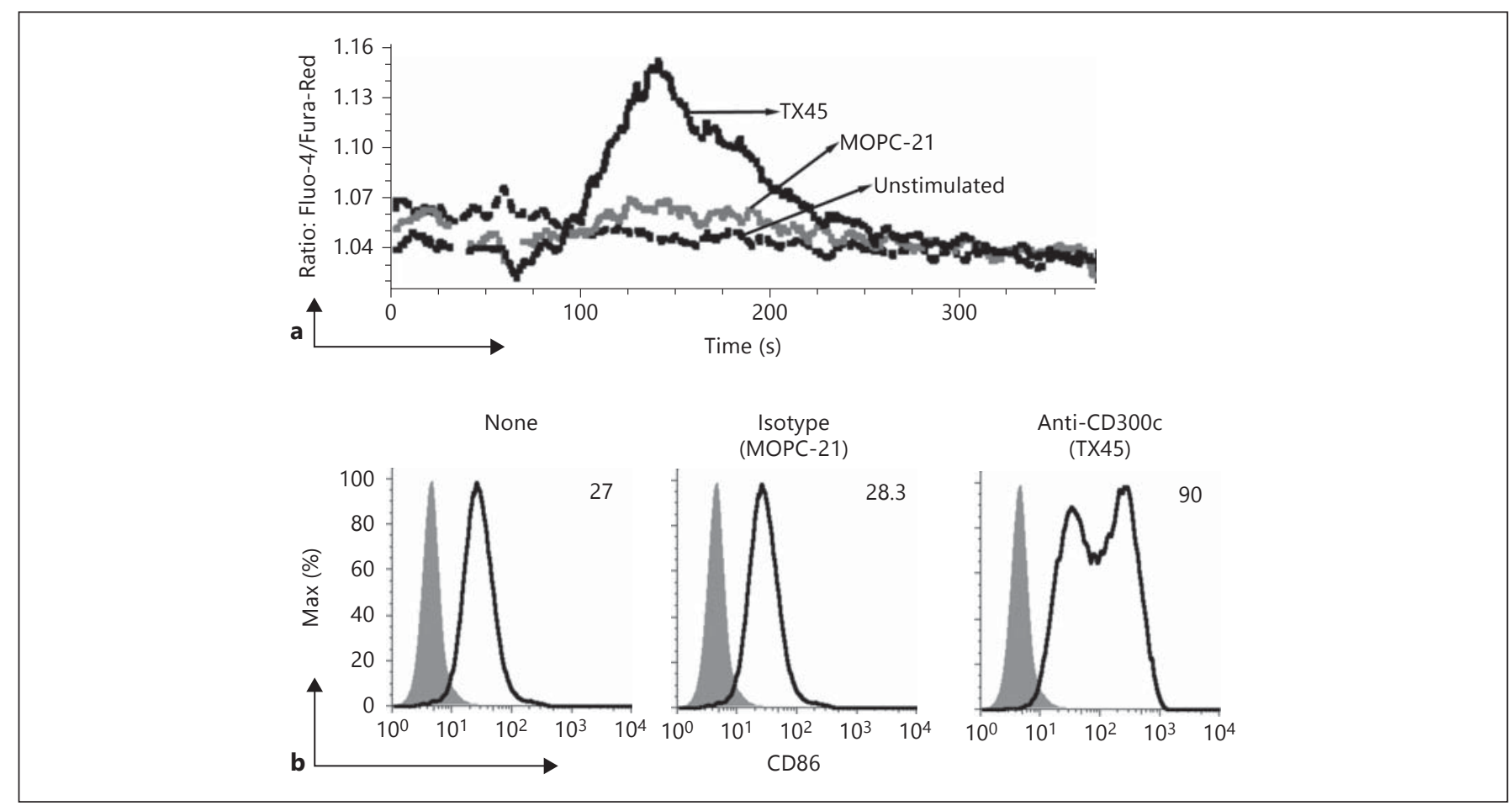

Fig. 4. Cross-linking of CD300c induces calcium mobilization and upregulation of CD86. a Enriched monocytes from human healthy donors were loaded with Fluo-4 and Fura-Red. To establish a baseline, monocytes were first acquired using a FACSCalibur for $30 \mathrm{~s}$ at which point the primary antibodies, anti-CD300c (clone TX45) or isotype-matched control (clone MOPC-21), were added. Then, at $60 \mathrm{~s}$, the primary antibodies were cross-linked with goat antimouse IgG F $\left(\mathrm{ab}^{\prime}\right)_{2}$ and fluorescence was measured. $\mathrm{Ca}^{2+}$ mobilization is expressed as the ratio of Fluo-4/Fura-Red as a function of time. Results are representative of 3 independent experiments.

\section{Discussion}

Monocytes have a very important role in both innate and adaptive immune responses, and their tasks are in part controlled and regulated by cell surface receptors that are able to deliver activating or inhibitory signals. The CD300 family of molecules regulates a large range of immune cell processes via their paired activating and inhibitory receptors $[9,20]$. Among these molecules, it has been shown that the activating members, CD300b and CD300e, and the inhibitory receptors, CD300a and CD300f, have a prominent role in regulating functions of human primary monocytes and monocytic cell lines $[15,16,19,34,35]$. In this article, we show that CD300c, another member of the CD300 family of receptors, is only expressed on monocytes within blood leukocytes. Furthermore, cross-linking of CD300c with an agonist b Enriched monocytes from human healthy donors were left either nonstimulated (None) or stimulated with plate-bound isotype control antibody, MOPC-21 (Isotype), and anti-CD300c monoclonal antibody, clone TX45 (TX45) for $24 \mathrm{~h}$. Then, cells were harvested, stained and analyzed by flow cytometry. Shaded histograms correspond to unstained cells and open histograms to the specific staining with anti-CD86 monoclonal antibody. Numbers indicate the values of the median fluorescence intensity. These results are representative of 3 independent experiments from 3 different healthy donors.

antibody led to the activation of monocytes, as shown by mobilization of intracellular calcium, upregulation of the costimulatory receptor CD86 and production of cytokines. We also show that the expression of CD300c on primary monocytes is regulated upon stimulation with TLR4 and TLR5 ligands, and that it has a costimulatory effect with LPS in the secretion of proinflammatory cytokines. Altogether, our results indicate that CD300c is an activating receptor expressed by human monocytes that may have a very important role during inflammation.

Elucidating the expression and function of human $\mathrm{CD} 300 \mathrm{c}$ in primary cells has been hampered by the unavailability of specific monoclonal antibodies. CD300a and $\mathrm{CD} 300 \mathrm{c}$ are paired receptors with more than $80 \%$ homology in the amino acid sequence of the extracellular domain [22], and the majority of available monoclonal 


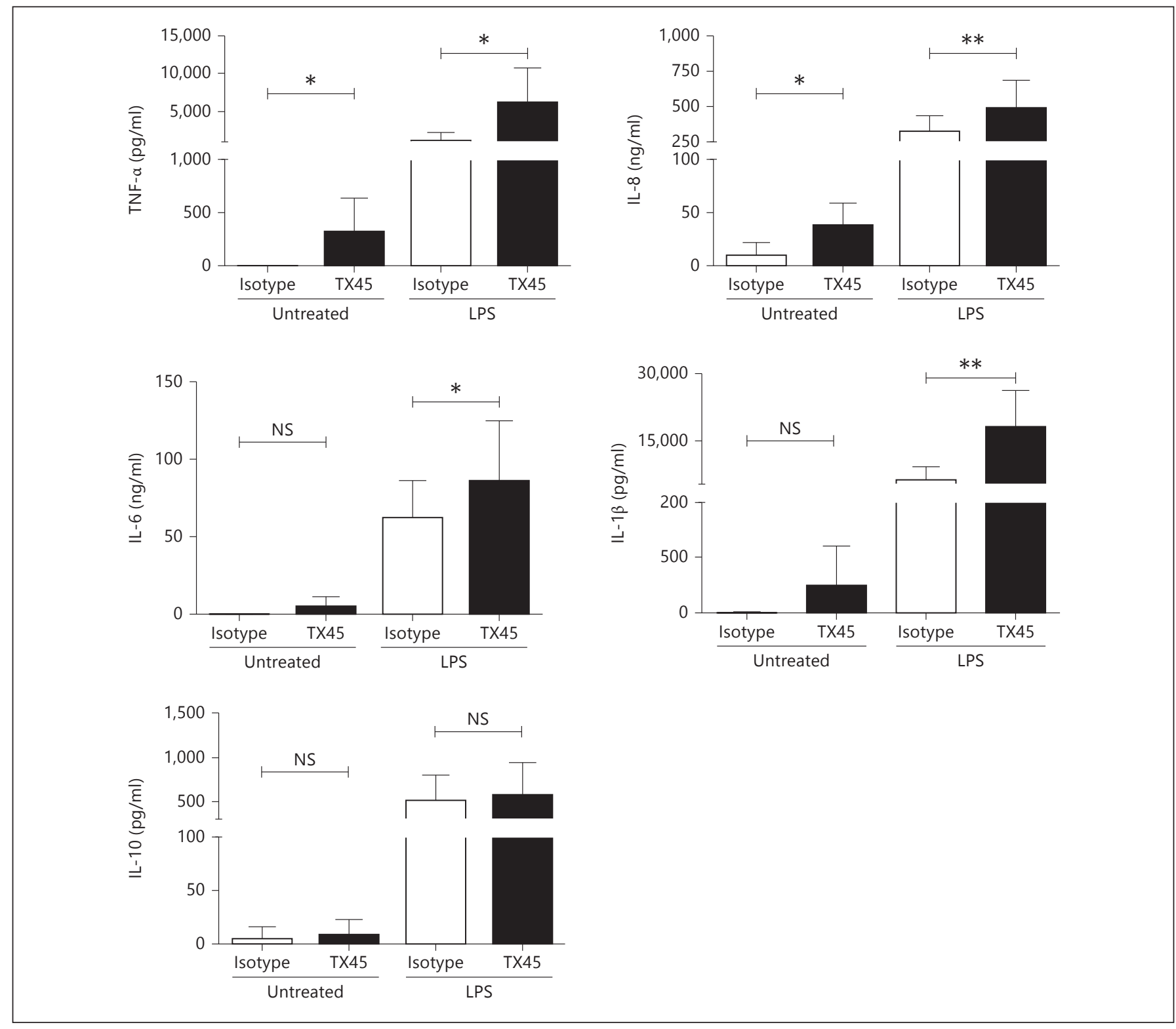

Fig. 5. Cross-linking of CD300c in monocytes induces the secretion of inflammatory cytokines. Freshly isolated monocytes from healthy donors were either stimulated with plate-bound isotypematched control antibody, MOPC-21 (empty bars), or with antiCD300c antibody, TX45 (black bars), in the absence (Untreated) or presence of LPS for $24 \mathrm{~h}$. Culture supernatants were harvested and tested for the secretion of human inflammatory cytokines using flow-cytometric bead analysis. The values on the y-axis correspond to the concentrations of cytokines: TNF- $\alpha$, IL- $1 \beta$ and IL-10 $(\mathrm{pg} / \mathrm{ml})$, and IL- 8 and IL-6 (ng/ml). Graph bars represent the average \pm SEM. Data are from 5 independent experiments.

cells. Although previous reports have described that CD300a and CD300c transcripts are expressed on lymphoid and myeloid cells, the cell surface expression of both receptors was not clarified. Our data demonstrate that CD300c is expressed exclusively on the surface of human monocytes, and also on other monocyte-derived cells like macrophages and DC. On the other hand, the 
binding of monoclonal antibodies that recognize both CD300a and CD300c, including clones E59.126 and TX49, to other cell types, such as T, B and NK cells and granulocytes $[22,23,27,28,30-32]$, suggest that what those antibodies are recognizing on these cells is probably CD300a but not CD300c. The latter has been suspected in some way because cross-linking experiments with anti$\mathrm{CD} 300 \mathrm{a} / \mathrm{c}$ monoclonal antibodies have been shown to deliver inhibitory signals in several cell types $[9,23,27,28$, $31,34]$.

The presence of $C D 300 c$ transcripts in other cell types, in addition to monocytes, raises the question of what mechanisms control the cell surface expression of this receptor. It is important to note that stimulation of cultured NK clones and of cultured primary CD4+ T cells with anti-CD300a/c monoclonal antibodies do not always induce a negative signal $[22,28]$, suggesting the possibility that in these circumstances, the monoclonal antibodies may also be recognizing CD300c. Clearly, further investigations are required in this regard to determine if cell culture and activation induces cell surface expression of CD300c. In our study, we report that both transcripts and cell surface expression of CD300c on monocytes were downregulated with LPS or flagellin treatment after $2 \mathrm{~h}$. On the other hand, the cell surface expression is significantly upregulated after $24 \mathrm{~h}$ of treatment, without any apparent increase in mRNA levels compared with nonstimulated monocytes. These results suggest that the regulation of CD300c surface expression on monocytes is regulated both at transcriptional and posttranscriptional level. The expression of other CD300 molecules has been shown to be regulated by posttranscriptional mechanisms. For example, mouse CD300b expression on neutrophils is regulated by the action of matrix metalloproteinases, and TLR4 stimulation increases the release of CD300b from the cell surface [36]. It has also been described that stimulation of human neutrophils with LPS and GM-CSF causes a rapid translocation of an intracellular pool of CD300a to the cell surface [23]. Our preliminary data suggest that matrix metalloproteinases may also have a role in the downregulation of $\mathrm{CD} 300 \mathrm{c}$ from the surface of monocytes after $2 \mathrm{~h}$ of stimulation with LPS or flagellin (data not shown).

Monocytes are recruited and activated at the sites of inflammation, secrete cytokines and upregulate the expression of costimulatory molecules that are important for optimal T-cell responses [37]. Similar to CD300e [16], we have also shown that cross-linking of CD300c with agonist antibodies induces mobilization of intracel- lular calcium, cytokine production and upregulation of CD86 expression on human monocytes. On the other hand, we could not see an upregulation of CD80 expression in our experimental settings. This could imply that $\mathrm{CD} 300 \mathrm{c}$ is a bona fide activating receptor, since it has been proposed that CD80 and CD86 have different functional identities, namely that CD86 is the main ligand for CD28, a T-cell costimulatory molecule, and CD80 is the major ligand for CD152, a T-cell inhibitory receptor [38]. Clearly, more studies are required to address this issue.

Similar to ligation of CD300e, engagement of CD300c with antibodies triggered the secretion of TNF- $\alpha$ and IL- 8 by monocytes. However, as opposed to CD300e [16], the cross-linking of CD300c further enhanced LPSmediated inflammatory cytokine production, while it did not increase the secretion of IL-10, emphasizing the proinflammatory role of CD300c. Nonetheless, it is very important to note that despite the fact that agonist monoclonal antibodies are helpful tools to functionally characterize cell surface receptors, data should be carefully interpreted for comparative analysis between different molecules, unless they are validated with their natural ligands. Recently, it has been published that certain CD300 molecules have the ability to functionally interact with lipids [10-12, 14]. Specifically, human CD300a has been shown to interact with phosphatidylserine and phosphatidylethanolamine, two lipids that are exposed on the outer leaflet of the plasma membrane of apoptotic and activated cells [14]. Although the natural ligand of human CD300c is unknown, given that CD300a and CD300c show more than 90\% homology in their IgV like domain, it would not be unreasonable to expect that CD300c may bind the same or structurally similar lipids to those that are recognized by CD300a. Further studies are required to identify the ligand of human $\mathrm{CD} 300 \mathrm{c}$ and to fully understand its physiological role.

In conclusion, here we show that the clone TX45 monoclonal antibody specifically recognizes CD300c. We demonstrate that this activating receptor is only expressed on the surface of human monocytes within peripheral blood leukocytes, and that its engagement results in the production of proinflammatory cytokines and upregulation of ligands for T-cell costimulatory receptors. Altogether, this suggests that CD300c may have a very important role in regulating different immune responses. 


\section{Acknowledgments}

The authors would like to thank Dr. Akira Shibuya for the generous gift of clone TX49 antibody. This work was funded by the intramural program of the Food and Drug Administration.

\section{Note Added in Proof}

Takahashi et al. [39] have also shown that human CD300c is expressed on the cell surface of monocytes and is able to deliver an activating signal after cross-linking with specific anti-CD300c antibodies.

\section{Disclosure Statement}

The authors declare no competing financial interests.

\section{References}

1 Soehnlein O, Lindbom L: Phagocyte partnership during the onset and resolution of inflammation. Nat Rev Immunol 2010;10:427-439.

2 Wong KL, Yeap WH, Tai JJ, Ong SM, Dang TM, Wong SC: The three human monocyte subsets: implications for health and disease. Immunol Res 2012;53:41-57.

-3 Brown D, Trowsdale J, Allen R: The LILR family: modulators of innate and adaptive immune pathways in health and disease. Tissue Antigens 2004;64:215-225.

4 Colonna M:TREMs in the immune system and beyond. Nat Rev Immunol 2003;3:445-453.

5 Humphrey MB, Lanier LL, Nakamura MC: Role of ITAM-containing adapter proteins and their receptors in the immune system and bone. Immunol Rev 2005;208:50-65.

-6 Moretta A, Bottino C, Vitale M, Pende D, Cantoni C, Mingari MC, Biassoni R, Moretta $\mathrm{L}$ : Activating receptors and coreceptors involved in human natural killer cell-mediated cytolysis. Annu Rev Immunol 2001;19:197223.

7 Borrego F, Kabat J, Kim DK, Lieto L, Maasho K, Pena J, Solana R, Coligan JE: Structure and function of major histocompatibility complex (MHC) class I specific receptors expressed on human natural killer (NK) cells. Mol Immunol 2002;38:637-660.

8 Ravetch JV, Lanier LL: Immune inhibitory receptors. Science 2000;290:84-89.

-9 Borrego F: The CD300 molecules: an emerging family of regulators of the immune system. Blood 2013;121:1951-1960.

10 Cannon JP, O’Driscoll M, Litman GW: Specific lipid recognition is a general feature of CD300 and TREM molecules. Immunogenetics 2012;64:39-47.

11 Choi SC, Simhadri VR, Tian L, Gil-Krzewska A, Krzewski K, Borrego F, Coligan JE: Cutting edge: mouse CD300f (CMRF-35like molecule-1) recognizes outer membrane-exposed phosphatidylserine and can promote phagocytosis. J Immunol 2011;187: 3483-3487.

12 Izawa K, Yamanishi Y, Maehara A, Takahashi M, Isobe M, Ito S, Kaitani A, Matsukawa T, Matsuoka T, Nakahara F, Oki T, Kiyonari H, Abe T, Okumura K, Kitamura T, Kitaura J:
The receptor LMIR3 negatively regulates mast cell activation and allergic responses by binding to extracellular ceramide. Immunity 2012;37:827-839.

13 Nakahashi-Oda C, Tahara-Hanaoka S, Shoji M, Okoshi Y, Nakano-Yokomizo T, Ohkohchi N, Yasui T, Kikutani H, Honda S, Shibuya K, Nagata S, Shibuya A: Apoptotic cells suppress mast cell inflammatory responses via the CD300a immunoreceptor. J Exp Med 2012;209:1493-1503.

14 Simhadri VR, Andersen JF, Calvo E, Choi SC, Coligan JE, Borrego F: Human CD300a binds to phosphatidylethanolamine and phosphatidylserine, and modulates the phagocytosis of dead cells. Blood 2012;119: 2799-2809.

15 Yamanishi Y, Kitaura J, Izawa K, Kaitani A, Komeno Y, Nakamura M, Yamazaki S, Enomoto Y, Oki T, Akiba H, Abe T, Komori T, Morikawa Y, Kiyonari H, Takai T, Okumura $\mathrm{K}$, Kitamura T: TIM1 is an endogenous ligand for LMIR5/CD300b: LMIR5 deficiency ameliorates mouse kidney ischemia/reperfusion injury. J Exp Med 2010;207:15011511.

16 Brckalo T, Calzetti F, Perez-Cabezas B, Borras FE, Cassatella MA, Lopez-Botet M: Functional analysis of the CD300e receptor in human monocytes and myeloid dendritic cells. Eur J Immunol 2010;40:722-732.

-17 Comas-Casellas E, Martinez-Barriocanal A, Miro F, Ejarque-Ortiz A, Schwartz S Jr, Martin $\mathrm{M}$, Sayos J: Cloning and characterization of CD300d, a novel member of the human CD300 family of immune receptors. J Biol Chem 2012;287:9682-9693.

18 Martinez-Barriocanal A, Comas-Casellas E, Schwartz S Jr, Martin M, Sayos J: CD300 heterocomplexes, a new and family-restricted mechanism for myeloid cell signaling regulation. J Biol Chem 2010;285: 41781-41794.

19 Martinez-Barriocanal A, Sayos J: Molecular and functional characterization of CD300b, a new activating immunoglobulin receptor able to transduce signals through two different pathways. J Immunol 2006;177:28192830 .
20 Gasiorowski RE, Ju X, Hart DN, Clark GJ: CD300 molecule regulation of human dendritic cell functions. Immunol Lett 2013;149: 93-100.

21 Clark GJ, Green BJ, Hart DN: The CMRF$35 \mathrm{H}$ gene structure predicts for an independently expressed member of an ITIM/ITAM pair of molecules localized to human chromosome 17. Tissue Antigens 2000;55:101-109.

22 Lankry D, Simic H, Klieger Y, Levi-Schaffer F, Jonjic S, Mandelboim O: Expression and function of CD300 in NK cells. J Immunol 2010; $185: 2877-2886$.

23 Alvarez Y, Tang X, Coligan JE, Borrego F: The CD300a (IRp60) inhibitory receptor is rapidly up-regulated on human neutrophils in response to inflammatory stimuli and modulates CD32a (FcgammaRIIa) mediated signaling. Mol Immunol 2008;45:253-258.

24 Bachelet I, Munitz A, Moretta A, Moretta L, Levi-Schaffer F: The inhibitory receptor IRp60 (CD300a) is expressed and functional on human mast cells. J Immunol 2005; 175 : 7989-7995.

25 DeBell KE, Simhadri VR, Mariano JL, Borrego F: Functional requirements for inhibitory signal transmission by the immunomodulatory receptor CD300a. BMC Immunol 2012;13:23.

$26 \mathrm{Ju}$ X, Zenke M, Hart DN, Clark GJ: CD300a/c regulate type I interferon and TNF-alpha secretion by human plasmacytoid dendritic cells stimulated with TLR7 and TLR9 ligands. Blood 2008;112:1184-1194.

27 Silva R, Moir S, Kardava L, Debell K, Simhadri VR, Ferrando-Martinez S, Leal M, Pena J, Coligan JE, Borrego F: CD300a is expressed on human B cells, modulates BCR-mediated signaling, and its expression is down-regulated in HIV infection. Blood 2011;117:5870-5880.

28 Simhadri VR, Mariano JL, Zhou Q, DeBell KE, Borrego F: Differential expression of CD300a/c on human TH1 and TH17 cells. BMC Immunol 2011;12:62.

29 Jackson DG, Hart DN, Starling G, Bell JI: Molecular cloning of a novel member of the immunoglobulin gene superfamily homologous to the polymeric immunoglobulin receptor. Eur J Immunol 1992;22:1157-1163. 
30 Green BJ, Clark GJ, Hart DN: The CMRF-35 $\mathrm{mAb}$ recognizes a second leukocyte membrane molecule with a domain similar to the poly Ig receptor. Int Immunol 1998;10:891-899.

-31 Cantoni C, Bottino C, Augugliaro R, Morelli L, Marcenaro E, Castriconi R, Vitale M, Pende D, Sivori S, Millo R, Biassoni R, Moretta L, Moretta A: Molecular and functional characterization of IRp60, a member of the immunoglobulin superfamily that functions as an inhibitory receptor in human NK cells. Eur J Immunol 1999;29:3148-3159.

-32 Narayanan S, Silva R, Peruzzi G, Alvarez Y, Simhadri VR, Debell K, Coligan JE, Borrego F: Human Th1 cells that express CD300a are polyfunctional and after stimulation up-regulate the T-box transcription factor eomesodermin. PLoS One 2010;5:e10636.
3 Akira S: Toll receptor families: structure and function. Semin Immunol 2004;16:1-2.

34 Kim EJ, Lee SM, Suk K, Lee WH: CD300a and CD300f differentially regulate the MyD88 and TRIF-mediated TLR signalling pathways through activation of SHP-1 and/or SHP-2 in human monocytic cell lines. Immunology 2012;135:226-235.

35 Wu Y, Chen Q, Pai T, Ross AC: All-trans-retinoic acid and Erk1/2 signaling synergistically regulate the expression of $\mathrm{CD} 300 \mathrm{~b}$ in human monocytic cells. Cell Immunol 2011;268:6878.

36 Yamanishi $\mathrm{Y}$, Takahashi M, Izawa K, Isobe M, Ito S, Tsuchiya A, Maehara A, Kaitani A, Uchida T, Togami K, Enomoto Y, Nakahara F, Oki T, Kajikawa M, Kurihara H, Kitamura T, Kitaura J: A soluble form of LMIR5/ CD300b amplifies lipopolysaccharide-induced lethal inflammation in sepsis. J Immunol 2012;189:1773-1779.
37 Serbina NV, Shi C, Pamer EG: Monocyte-mediated immune defense against murine Listeria monocytogenes infection. Adv Immunol 2012;113:119-134.

38 Sansom DM, Manzotti CN, Zheng Y: What's the difference between CD80 and CD86? Trends Immunol 2003;24:314-319.

39 Takahashi M, Izawa K, Kashiwakura J, Yamanishi Y, Enomoto Y, Kaitani A, Maehara A, Isobe $\mathrm{M}$, Ito $\mathrm{S}$, Matsukawa $\mathrm{T}$, Nakahara $\mathrm{F}$, Oki T, Kajikawa M, Ra C, Okayama Y, Kitamura T, Kitaura J: Human CD300C delivers an $\mathrm{F}_{\mathrm{c}}$ receptor-gamma-dependent activating signal in mast cells and monocytes and differs from CD300A on ligand recogntiion. J Biol Chem 2013;288:7662-7675. 